\title{
Derivative and antiderivative operators and the size of complex domains
}

\author{
by Luis Bernal-GonzÁlez (Sevilla)
}

Dedicated to the memory of Prof. A. de Castro Brzezicki

\begin{abstract}
We prove some conditions on a complex sequence for the existence of universal functions with respect to sequences of certain derivative and antiderivative operators related to it. These operators are defined on the space of holomorphic functions in a complex domain. Conditions for the equicontinuity of those sequences are also studied. The conditions depend upon the size of the domain.
\end{abstract}

1. Introduction and notation. We denote by $\mathbb{C}$ the complex plane and by $G$ a simply connected domain, i.e., $G \subset \mathbb{C}, G$ is nonempty, open and connected, and its complement with respect to the extended plane is also connected. $H(G)$ denotes, as usual, the space of holomorphic functions in $G$, endowed with the topology of uniform convergence on compact subsets.

$B(a, r)(\bar{B}(a, r))$ is the euclidean open (closed, respectively) disk with center $a$ and radius $r$. We agree that $B(a,+\infty)=\mathbb{C}$. $\mathbb{N}$ is the set of positive integers. If $g$ is a complex function defined on a subset $A \subset \mathbb{C}$, we write $\|g\|_{A}=\sup _{z \in A}|g(z)|$. If $A, B \subset \mathbb{C}$, then $d(A, B)$ will stand for the infimum of the distances $|a-b|(a \in A, b \in B)$ and the diameter of $A$ is $\operatorname{diam}(A)=\sup \{|a-b|: a, b \in A\}$. We adopt the conventions $d(a, \emptyset)=+\infty$ $(a \in \mathbb{C}), 1 /+\infty=0$ and $1 / 0=+\infty$. We define the circumscribed radius of $G$ as

$$
\begin{aligned}
R(G) & =\inf _{a \in \mathbb{C}} \sup _{b \in G}|a-b| \\
& =\inf \{r>0: \text { there is an open disk } B \text { of radius } r \text { with } G \subset B\},
\end{aligned}
$$

and the inscribed radius of $G$ as

1991 Mathematics Subject Classification: Primary 30E10, 47B38.

Key words and phrases: universal function, equicontinuous sequence, derivative operator, antiderivative operator, MacLane's theorem, size of a domain. 


$$
\begin{aligned}
\varrho(G) & =\sup _{b \in G} \inf _{a \notin G}|a-b| \\
& =\sup \{r>0: \text { there is an open disk } B \text { of radius } r \text { with } B \subset G\} .
\end{aligned}
$$

The numbers $\operatorname{diam}(G), R(G)$ and $\varrho(G)$ describe, elementarily, the "size" of the domain $G$. If we fix a point $a \in G$, we define

$$
\Delta(G)=\sup _{z \in G} \inf \{r>0: a \text { is in the connected component }
$$

$$
\text { of } B(z, r) \cap G \text { containing } z\} \text {. }
$$

It is easy to check the following:

(a) $0<\varrho(G) \leq(1 / 2) \operatorname{diam}(G) \leq R(G) \leq(\sqrt{3} / 2) \operatorname{diam}(G) \leq \infty$.

(b) $G$ is bounded if and only if $\operatorname{diam}(G)$ is finite if and only if $R(G)$ is finite if and only if $\Delta(G)$ is finite.

(c) If $G$ is bounded, then $\varrho(G)=R(G)$ if and only if $G$ is an open disk.

(d) $d(a, \mathbb{C} \backslash G) \leq \sup _{z \in G}|z-a| \leq \Delta(G) \leq \operatorname{diam}(G)$.

(e) If $G$ is starlike with respect to $a$, then $\Delta(G)=\sup _{z \in G}|z-a|$.

(f) $d(a, \mathbb{C} \backslash G)=\Delta(G)$ if and only if $G$ is $\mathbb{C}$ or an open disk with center $a$. In this last case, the common value is the radius of $G$.

The set of polynomials is dense in $H(G)$ by Runge's theorem [12, pp. 288291]. $H(G)$ is a second-countable Fréchet space. A topological space $X$ is a Baire space if and only if the intersection of a countable family of open dense subsets is also dense. Baire's category theorem asserts that each completely metrizable topological space is a Baire space. Consequently, $H(G)$ is a Baire space. In a Baire space $X$, a subset is residual when it contains a dense $G_{\delta}$ subset of $X$. Such a subset is "very large" in $X$. For this see, for instance, [8, pp. 213-214 and 238] and [11, pp. 40-41].

We use a very general notion of universality, which can be found in [6], namely: Let $X$ and $Y$ be topological spaces and $T_{n}: X \rightarrow Y(n \in \mathbb{N})$ a sequence of continuous mappings. Then an element $x \in X$ is called $\left\{T_{n}\right\}_{n=1^{-}}^{\infty}$ universal if its orbit $\left\{T_{n}(x): n \in \mathbb{N}\right\}$ is dense in $Y$. Several versions of the following result can be found in [5], where universal vectors are called hypercyclic. Its proof is an application of Baire's theorem.

THEOREM A. Let $X$ be a linear topological space that is a Baire space, $Y$ a linear topological space that is second-countable, $D \subset X$ dense in $X, D^{\prime} \subset Y$ dense in $Y$ and $T_{n}: X \rightarrow Y(n \in \mathbb{N})$ a countable family of continuous linear mappings satisfying the following condition:

(A) For every $d \in D$ and every $d^{\prime} \in D^{\prime}$ there exist a sequence $\left\{x_{p}\right.$ : $p \in \mathbb{N}\} \subset X$ and positive integers $n_{1}<n_{2}<\ldots$ such that $x_{p} \rightarrow 0$, $T_{n_{p}}(d) \rightarrow 0$ and $T_{n_{p}}\left(x_{p}\right) \rightarrow d^{\prime}(p \rightarrow \infty)$.

Then the subset of $\left\{T_{n}\right\}_{n=1}^{\infty}$-universal vectors $x \in X$ is residual in $X$. 
In addition, we point out that if $X$ and $Y$ are two nontrivial metric linear spaces and $\left\{T_{n}: n \in \mathbb{N}\right\}$ is an equicontinuous family of linear operators from $X$ into $Y$, then the orbit $\left\{T_{n}(x): n \in \mathbb{N}\right\}$ of each $x \in X$ is bounded; so there is no $\left\{T_{n}\right\}_{n=1}^{\infty}$-universal vector in $X$.

In this paper derivative and antiderivative operators will be considered. These are defined as follows. The derivative operators are

$$
D^{n}: H(G) \ni f \mapsto f^{(n)} \in H(G) \quad(n \in \mathbb{N}) .
$$

If a point $a \in G$ is fixed, the antiderivative operators are

$$
I^{n}: H(G) \ni f \mapsto \int_{a}^{z} I^{n-1} f(t) d t \in H(G) \quad(n \in \mathbb{N}),
$$

with $I^{0} f=f$, the integral being taken along any rectifiable arc in $G$ joining $a$ to $z$. These operators are linear and continuous. Further, we have

$$
I^{n} f(z)=\frac{1}{(n-1) !} \int_{a}^{z}(z-t)^{n-1} f(t) d t \quad(\forall n \in \mathbb{N}, \forall f \in H(G), \forall z \in G) .
$$

MacLane's theorem [10] states that there exists $f \in H(\mathbb{C})$ such that the orbit $\left\{f^{(n)}: n \in \mathbb{N}\right\}$ is dense in $H(\mathbb{C})$ or, equivalently, $f$ is $\left\{D^{n}\right\}_{n=1^{-}}^{\infty}$ universal in $H(\mathbb{C})$. This theorem is also proved in [1]. S. M. Duios Ruis [3] has proved that, indeed, there is a residual set of such functions. Furthermore, R. M. Gethner and J. H. Shapiro [4] and K. G. Grosse-Erdmann [6, Satz 2.2.8] have derived the same result for every simply connected domain (see also [7] for a sharp result on growth of $\left\{D^{n}\right\}_{n=1}^{\infty}$-universal entire functions). Trivially, there cannot be any $\left\{I_{n}\right\}_{n=1}^{\infty}$-universal function $f \in H(G)$, because $I^{n} f(a)=0(\forall n \in \mathbb{N})$. Nevertheless, it is shown in [2] that there exists a sequence $\left\{C_{n}\right\}_{n=1}^{\infty} \subset \mathbb{C}$ with the following property: For every entire function $\phi$ the set $\left\{Q_{n}(z)=I^{n} \phi(z)+\sum_{j=0}^{n-1}\left(C_{n-j} / j !\right) z^{j}: n \in \mathbb{N}\right\}$ is dense in $H(\mathbb{C})$ (note that the coefficients $C_{n}$ 's do not depend upon $\phi$ ). This has been extended by W. Luh [9] to functions $\phi$ which are holomorphic in an open set with simply connected components.

All these results will be generalized and strengthened in this paper, by inserting a multiplicative complex sequence $\left\{c_{n}\right\}_{n=1}^{\infty}$. In addition, the equicontinuity of generalized derivative and antiderivative operators will be studied. The size of the domain $G$ will play an important role. We associate with $\left\{c_{n}\right\}_{n=1}^{\infty}$ the numbers $\alpha, \beta \in[0, \infty]$ given by

$$
\begin{aligned}
& \alpha=\limsup _{n \rightarrow \infty}\left(n !\left|c_{n}\right|\right)^{1 / n}=\frac{1}{e} \limsup _{n \rightarrow \infty}\left(n\left|c_{n}\right|^{1 / n}\right), \\
& \beta=\limsup _{n \rightarrow \infty}\left(\frac{\left|c_{n}\right|}{n !}\right)^{1 / n}=e \limsup _{n \rightarrow \infty} \frac{\left|c_{n}\right|^{1 / n}}{n} .
\end{aligned}
$$

The equalities of limsup's hold because of Stirling's formula. 
2. Derivative operators. First, we ask if there exists $f \in H(G)$ such that the orbit $\left\{c_{n} f^{(n)}: n \in \mathbb{N}\right\}$ is dense. The following result strengthens MacLane's theorem substantially; for this, choose $c_{n}=1(\forall n \in \mathbb{N})$.

THEOREM 1. If $R(G) \leq \alpha$, then the subset of $f \in H(G)$ such that the orbit $\left\{c_{n} f^{(n)}: n \in \mathbb{N}\right\}$ is dense in $H(G)$ is residual.

P r o o f. By the definition of $R(G)$, there is $a \in \mathbb{C}$ such that $|z-a|<R(G)$ $\forall z \in G$. Then $\sup _{z \in K}|z-a|<R(G)$ for every compact subset $K \subset G$. Apply Theorem A to $X=Y=H(G), D=D^{\prime}=\{$ polynomials $\}$ and $T_{n}=c_{n} D^{n}(n \in \mathbb{N})$. Fix two polynomials $P, Q$ with $Q(z)=\sum_{j=0}^{m} a_{j}(z-a)^{j}$. By hypothesis, there is a sequence of positive integers $n_{1}<n_{2}<\ldots$ with $c_{n_{p}} \neq 0(\forall p \in \mathbb{N})$ and $\lim _{p \rightarrow \infty}\left(n_{p} !\left|c_{n_{p}}\right|\right)^{1 / n_{p}} \geq R(G)$. Thus

$$
\lim _{p \rightarrow \infty} \frac{\left(\sup _{z \in K}|z-a|\right)^{n_{p}}}{n_{p} !\left|c_{n_{p}}\right|}=0 .
$$

Define $f_{p}$ by

$$
f_{p}(z)=\sum_{j=0}^{m} \frac{j ! a_{j}(z-a)^{j+n_{p}}}{\left(j+n_{p}\right) ! c_{n_{p}}} .
$$

Then $T_{n_{p}}(P)=0$ whenever $n_{p}>$ degree $(P), T_{n_{p}}\left(f_{p}\right)=Q(\forall p \in \mathbb{N})$ and, by $(1), f_{p} \rightarrow 0(p \rightarrow \infty)$ uniformly on compact subsets of $G$. So, condition (A) of Theorem A is evidently satisfied and the subset of functions which are $\left\{c_{n} D^{n}\right\}_{n=1}^{\infty}$-universal is residual. The theorem is proved.

Now, we give a necessary condition for the existence of $\left\{c_{n} D^{n}\right\}_{n=1^{-}}^{\infty}$ universal functions.

THEOREM 2. If there is $f \in H(G)$ whose orbit $\left\{c_{n} f^{(n)}: n \in \mathbb{N}\right\}$ is dense in $H(G)$, then $\varrho(G) \leq \alpha$.

Proof. Assume by way of contradiction that $\alpha<\varrho(G)$. Fix $r \in$ $(\alpha, \varrho(G))$. From the definition of $\varrho(G)$, there exists $b \in G$ such that $\bar{B}(b, r) \subset$ $G$. By assumption, $\lim _{n \rightarrow \infty} n !\left|c_{n}\right| / r^{n}=0$ and, because of Cauchy's inequalities,

$$
\left|c_{n} f^{(n)}(b)\right| \leq\left|c_{n}\right| \frac{n !\|f\|_{\bar{B}(b, r)}}{r^{n}} \rightarrow 0 \quad(n \rightarrow \infty) .
$$

Then the sequence $\left\{c_{n} f^{(n)}(b)\right\}_{n=1}^{\infty}$ is bounded, and so $\left\{c_{n} f^{(n)}\right\}_{n=1}^{\infty}$ cannot approximate the constant function $g(z)=1+\sup _{n \in \mathbb{N}}\left|c_{n} f^{(n)}(b)\right|$ on the compact set $K=\{b\}$. This is a contradiction.

COROLlary 1. If $B$ is an open disk, then the following statements are equivalent:

(a) The subset of $f \in H(B)$ such that the orbit $\left\{c_{n} f^{(n)}: n \in \mathbb{N}\right\}$ is dense in $H(B)$ is residual. 
(b) There is $f \in H(B)$ whose orbit $\left\{c_{n} f^{(n)}: n \in \mathbb{N}\right\}$ is dense in $H(B)$.

(c) $\operatorname{radius}(B) \leq \alpha$.

Next, the following two results furnish a necessary and sufficient condition for the equicontinuity. We can drop the hypothesis of simple connectedness. We distinguish the cases $G \neq \mathbb{C}, G=\mathbb{C}$.

THEOREM 3. If $G \subset \mathbb{C}$ is an arbitrary domain (simply connected or not) different from $\mathbb{C}$, then the family of operators $\mathcal{F}=\left\{c_{n} D^{n}: n \in \mathbb{N}\right\}$ is equicontinuous if and only if $\alpha=0$.

Proof. Assume that $\alpha=0$. Fix a basic neighborhood of the origin of $H(G)$, say $V(\varepsilon, K)=\{f \in H(G):|f(z)| \leq \varepsilon \forall z \in K\}$, where $\varepsilon>0$ and $K \subset G$ is compact. We must find $\delta>0$ and a compact subset $L \subset G$ satisfying

$$
\bigcup_{n \in \mathbb{N}} c_{n} D^{n}(V(\delta, K)) \subset V(\varepsilon, K)
$$

We have $\lim _{n \rightarrow \infty}\left(n !\left|c_{n}\right|\right)^{1 / n}=0$, by hypothesis. Pick a cycle $\gamma \subset G \backslash K$ (see $[12$, p. 287]) such that Cauchy's formula

$$
f^{(n)}(z)=\frac{n !}{2 \pi i} \oint_{\gamma} \frac{f(t)}{(t-z)^{n+1}} d t
$$

holds for $n \in \mathbb{N}, z \in K$ and $f \in H(G)$. The constant $M=\sup _{n \in \mathbb{N}} n !\left|c_{n}\right| /$ $d(K, \gamma)^{n}$ is finite. Choose $L=\gamma$ and $\delta=2 \pi \varepsilon d(K, \gamma) /(1+M \operatorname{length}(\gamma))$. If $n \in \mathbb{N}, f \in V(\delta, L)$ and $z \in K$, we have

$$
\left|c_{n} f^{(n)}(z)\right|=\left|\frac{n ! c_{n}}{2 \pi i} \oint_{\gamma} \frac{f(t)}{(t-z)^{n+1}} d t\right| \leq \frac{n !\left|c_{n}\right| \operatorname{length}(\gamma)\|f\|_{\gamma}}{2 \pi d(K, \gamma)^{n+1}} \leq \varepsilon .
$$

Consequently, (2) is satisfied and this proves that $\mathcal{F}$ is equicontinuous.

It remains to show that if $\alpha>0$ then $\mathcal{F}$ is not equicontinuous. Fix a point $a$ in $G$ and put $R:=d(a, \mathbb{C} \backslash G)=|a-b|$, where $b \in \mathbb{C} \backslash G$. Fix $r$ with $0<r<R$ and $R-r<\alpha$. Put $K=\bar{B}(a, r)$ and $\varepsilon=1$. Let $L$ be any compact subset of $G$ and $\delta$ a positive number. Let $m>0$ be so small that $m /|z-a|<\delta$, if $z \in L \cup K$. Then the function $f(z):=m /(z-a)$ belongs to $V(\delta, L)$ and $\sup \left\{\left|c_{n} f^{(n)}\right|: z \in K\right\}=n !\left|c_{n}\right| m /|R-r|^{n+1}$, so that $\sup \left\{n !\left|c_{n}\right| m /|R-r|^{n+1}: n \in \mathbb{N}\right\}=\infty$. Therefore

$$
\bigcup_{n \in \mathbb{N}} c_{n} D^{n}(V(\delta, L)) \not \subset V(1, K)
$$

which implies that $\mathcal{F}$ is not equicontinuous.

The condition of equicontinuity and the results of Theorems 1 and 2 can be stated jointly in the case $G=\mathbb{C}$. 
THEOREM 4. The following properties are equivalent:

(a) The sequence $\left\{\left(n !\left|c_{n}\right|\right)^{1 / n}\right\}_{n=1}^{\infty}$ is bounded.

(b) The sequence $\left\{n\left|c_{n}\right|^{1 / n}\right\}_{n=1}^{\infty}$ is bounded.

(c) There is no entire function $f$ such that $\left\{c_{n} f^{(n)}\right\}_{n=1}^{\infty}$ is dense in $H(\mathbb{C})$.

(d) The set of entire functions $f$ such that $\left\{c_{n} f^{(n)}\right\}_{n=1}^{\infty}$ is dense in $H(\mathbb{C})$ is not residual in $H(\mathbb{C})$.

(e) The family of operators $\left\{c_{n} D^{n}: n \in \mathbb{N}\right\}$ on $H(\mathbb{C})$ is equicontinuous.

Proof. Obviously, $R(\mathbb{C})=\varrho(\mathbb{C})=\infty$. Moreover, $\alpha$ is finite if and only if the sequence $\left\{\left(n !\left|c_{n}\right|\right)^{1 / n}\right\}_{n=1}^{\infty}$ is bounded. The equivalence of (a) and (b) is due to Stirling's formula. (a) implies (c) by Theorem 2. It is trivial that (c) implies (d). (d) implies (a) by Theorem 1. Therefore (a)-(d) are equivalent. It is evident that (e) implies (c). Hence, the unique thing to prove is that (a) implies (e). But we can follow the first part of the proof of Theorem 3 step by step with the sole exception that we may choose the cycle $\gamma$ in such a way that $\sup _{n \in \mathbb{N}}\left(n !\left|c_{n}\right|\right)^{1 / n} \leq d(K, \gamma)$ (note that this would not be possible if $G \neq \mathbb{C}$, except that $c_{n}=0 \forall n \in \mathbb{N}$ ). Then we obtain (2) by choosing $L=\gamma$ an $\delta=2 \pi \varepsilon d(K, \gamma) /$ length $(\gamma)$.

3. Antiderivative operators. Recall that, in the definition of $I^{n}(n \in$ $\mathbb{N}$ ), a point $a \in G$ has been fixed. We furnish a sufficient condition on the sequence $\left\{c_{n}\right\}_{n=1}^{\infty}$ in order to get a certain property of $\left\{c_{n} I^{n}\right\}_{n=1}^{\infty}$-universality, which generalizes that given in Section 1 (note that $\beta=0$ if $c_{n}=1 \forall n \in \mathbb{N}$ ). It turns also to be a sufficient condition for the equicontinuity of the family $\left\{c_{n} I^{n}: n \in \mathbb{N}\right\}$.

Lemma. If $\beta \leq 1 / \Delta(G)$ and $f \in H(G)$, then $\left\{c_{n} I^{n} f\right\}_{n=1}^{\infty}$ converges to zero uniformly on compact subsets.

Pro of. Let $z \in G$. From the definition of $\Delta(G), a$ lies in the connected component of $B(z, \Delta(G)) \cap G$ containing $z$. Then there exists a rectifiable $\operatorname{arc} \gamma_{z} \subset G$ joining $a$ to $z$ such that $|z-t|<\Delta(G)\left(\forall t \in \gamma_{z}\right)$. Fix a compact set $K \subset G$. Then we may choose a compact set $L$ and the $\operatorname{arcs}$ $\gamma_{z}$ in such a way that $\bigcup_{z \in K} \gamma_{z} \subset L \subset G, \sup _{z \in K}$ length $\left(\gamma_{z}\right)=S<\infty$ and $|z-t| \leq M=$ a constant $<\Delta(G)\left(\forall t \in \gamma_{z}, \forall z \in K\right)$. Fix $M_{1} \in(M, \Delta(G))$. Since $\beta \leq 1 / \Delta(G)$, there is $n_{0} \in \mathbb{N}$ such that $\left|c_{n}\right| /(n-1) ! \leq 1 / M_{1}^{n-1}$ whenever $n \geq n_{0}$. If $z \in K, n \geq n_{0}$ and $f \in H(G)$, we obtain

$$
\begin{aligned}
\left|c_{n} I^{n} f(z)\right| & =\left|\frac{c_{n}}{(n-1) !} \int_{\gamma_{z}}(z-t)^{n-1} f(t) d t\right| \leq \frac{\left|c_{n}\right|}{(n-1) !} M^{n-1}\|f\|_{L} \operatorname{length}\left(\gamma_{z}\right) \\
& \leq S\|f\|_{L}\left(M / M_{1}\right)^{n-1} \rightarrow 0 \quad(n \rightarrow \infty) .
\end{aligned}
$$

Thus $\lim _{n \rightarrow \infty}\left\|c_{n} I^{n} f\right\|_{K}=0$, as required. 
THEOREM 5. Assume that $\beta \leq 1 / \Delta(G)$. Then there exists a sequence $\left\{C_{n}\right\}_{n=1}^{\infty} \subset \mathbb{C}$ such that for every $f \in H(G)$ the sequence $\left\{Q_{n}(z)=c_{n} I^{n} f(z)\right.$ $\left.+\sum_{j=0}^{n-1}\left(C_{n-j} / j !\right) z^{j}: n \in \mathbb{N}\right\}$ has the following properties:

(a) $\left\{Q_{n}(z): n \in \mathbb{N}\right\}$ is dense in $H(G)$.

(b) For every compact set $B \subset G$ with connected complement and every function $g$ which is continuous on $B$ and holomorphic in the interior of $B$, there is a subsequence of $\left\{Q_{n}(z): n \in \mathbb{N}\right\}$ converging to $g$ uniformly on $B$.

(c) For every Lebesgue-measurable set $E \subset G$ and every Lebesguemeasurable function $g: E \rightarrow \mathbb{C} \cup\{\infty\}$, there is a subsequence of $\left\{Q_{n}(z)\right.$ : $n \in \mathbb{N}\}$ converging almost everywhere to $g$ on $E$.

Proof. We apply the mentioned result of Luh [9] to the domain $G$ and the function $\phi=0$. We obtain that there is a sequence $\left\{C_{n}\right\}_{n=1}^{\infty} \subset \mathbb{C}$ such that the set $\left\{H_{n}\right\}_{n=1}^{\infty}$ given by $H_{n}(z)=\sum_{j=0}^{n-1}\left(C_{n-j} / j !\right) z^{j}(n \in \mathbb{N})$ is dense in $H(G)$. By the lemma, $\left\{c_{n} I^{n} f\right\}_{n=1}^{\infty}$ tends to zero in $H(G)$. Thus $\left\{Q_{n}=c_{n} I^{n} f+H_{n}\right\}_{n=1}^{\infty}$ is also dense in $H(G)$. This proves (a). Finally, (b) and (c) are straightforward consequences of [9, Lemma 3].

THeOREm 6. (a) If $\beta \leq 1 / \Delta(G)$, then the family of operators $\left\{c_{n} I^{n}\right.$ : $n \in \mathbb{N}\}$, defined on $H(G)$, is equicontinuous.

(b) If $\beta>1 / d(a, \mathbb{C} \backslash G)$, then the above family is not equicontinuous.

Proof. (a) As in the proof of Theorem 3, fix a basic neighborhood of the origin $V(\varepsilon, K)$, where $\varepsilon>0$ and $K \subset G$ is compact. If one looks at the proof of the above lemma, the following is readily seen:

$$
\left|c_{n} I^{n} f(z)\right| \leq S \delta\left(M / M_{1}\right)^{n-1} \leq S \delta=\varepsilon
$$

whenever $\delta=\varepsilon / S, n \geq n_{0}, z \in K$ and $\|f\|_{L} \leq \delta$. Therefore

$$
\bigcup_{n \geq n_{0}} c_{n} I^{n}(V(\delta, L)) \subset V(\varepsilon, K) .
$$

This implies that the family $\left\{c_{n} I^{n}: n \geq n_{0}\right\}$ is equicontinuous. Therefore $\left\{c_{n} I^{n}: n \in \mathbb{N}\right\}$ is equicontinuous.

(b) Assume that $\beta>1 / d(a, \mathbb{C} \backslash G)$. Fix $\mu, \nu$ with $1 / d(a, \mathbb{C} \backslash G)<\mu<$ $\nu<\beta$. Suppose that $\delta>0$ and $L \subset G$ is compact. Consider the compact set $K=\{z:|z-a|=1 / \mu\}$. There is $m \in \mathbb{N}$ with $\delta(\nu / \mu)^{m}>1$ and $\left|c_{m}\right|>m ! \nu^{m}$. The constant function $f(z)=\delta$ is obviously in $V(\delta, L)$. If $z \in K$ we have

$$
\left|c_{m} I^{m} f(z)\right|=\left|\delta c_{m} \frac{(z-a)^{m}}{m !}\right|=\left|\frac{\delta c_{m}}{m ! \mu^{m}}\right| \geq \delta\left(\frac{\nu}{\mu}\right)^{m}>1,
$$

so $c_{m} I^{m}(V(\delta, L))$ is not included in $V(1, K)$. Hence $\left\{c_{n} I^{n}: n \in \mathbb{N}\right\}$ is not equicontinuous. 
Corollary 2. (a) If $G=\mathbb{C}$, then the family of operators $\left\{c_{n} I^{n}: n \in \mathbb{N}\right\}$ is equicontinuous if and only if $\beta=0$.

(b) If $G$ is an open disk with center a, then the family of operators $\left\{c_{n} I^{n}: n \in \mathbb{N}\right\}$ is equicontinuous if and only if $\beta \leq 1 / \operatorname{radius}(G)$.

The author would like to thank the referee for helpful comments and suggestions.

\section{References}

[1] C. Blair and L. A. Rubel, A universal entire function, Amer. Math. Monthly 90 (1983), 331-332.

[2] - - - A triply universal entire function, Enseign. Math. 30 (1984), 269-274.

[3] S. M. Duios Ruis, Universal functions of the structure of the space of entire functions, Soviet Math. Dokl. 30 (1984), 713-716.

[4] R. M. Gethner and J. H. Shapiro, Universal vectors for operators on spaces of holomorphic functions, Proc. Amer. Math. Soc. 100 (1987), 281-288.

[5] G. Godefroy and J. H. Shapiro, Operators with dense, invariants, cyclic vector manifolds, J. Funct. Anal. 98 (1991), 229-269.

[6] K. G. Grosse-Erdmann, Holomorphe Monster und universelle Funktionen, Mitt. Math. Sem. Giessen 176 (1987)

[7] - On the universal functions of G. R. MacLane, Complex Variables Theory Appl. 15 (1990), 193-196.

[8] J. Horváth, Topological Vector Spaces, Vol. 1, Addison-Wesley, Reading, 1966.

[9] W. Luh, Approximation by antiderivatives, Constr. Approx. 2 (1986), 179-187.

[10] G. R. MacLane, Sequences of derivatives and normal families, J. Analyse Math. 2 (1952), $72-87$.

[11] J. C. Oxtoby, Measure and Category, 2nd ed., Springer, New York, 1980.

[12] W. Rudin, Real and Complex Analysis, 2nd ed., Tata McGraw-Hill, Faridabad, 1974 .

DEPARTAMENTO DE ANÁLISIS MATEMÁTICO

FACULTAD DE MATEMÁTICAS, APDO. 1160

AVENIDA REINA MERCEDES

41080 SEVILLA, SPAIN 